\title{
Personalized embryo transfer after endometrial receptivity array test in patients with recurrent unexplained implantation failure
}

\author{
Nagwan Ahmed Bahgat ${ }^{1 *}$, Waleed Said ${ }^{2}$
}

\begin{abstract}
${ }^{1}$ Department of Obstetrics and Gynecology, Mansoura University Hospital, Mansoura Faculty of Medicine, Egypt ${ }^{2}$ Health Plus Fertility Center, Abu Dhabi, UAE
\end{abstract}

Received: 20 January 2022

Revised: 04 February 2022

Accepted: 05 February 2022

\section{*Correspondence:}

Dr. Nagwan Ahmed Bahgat,

E-mail: nagwanivm@yahoo.com

Copyright: (c) the author(s), publisher and licensee Medip Academy. This is an open-access article distributed under the terms of the Creative Commons Attribution Non-Commercial License, which permits unrestricted non-commercial use, distribution, and reproduction in any medium, provided the original work is properly cited.

\begin{abstract}
Background: Unexplained recurrent implantation failure is a devastating situation for both patients and the doctor treating them, with transfer of high grade euploid embryos this situation became more related to the endometrial receptivity and the interaction between the embryo and the endometrium. Till now the best way of detecting endometrial receptivity was through endometrial receptivity array of gene in endometrial tissue.

Methods: A retrospective study was carried out in large IVF center in Abu Dhabi in period from 2017-2021. Patients included in the study were infertile patients with age limit of 43 years old with history of repeated IVF failure after multiple transfer trials of high grade embryos. All patients had ERA test then frozen embryo transfer of Euploid high grade embryos obtained through stimulated cycle of each patient according to Era test results.

Results: 45 patients included in our study. Patients divided into 2 major groups according to Era test result. First group included patients with receptive endometrium. The second group was the patients with displaced window of implantation. Patients with receptive endometrium were $12(26.7 \%)$ and the displaced window of implantation was found in 33 patients $(73.3 \%)$. Higher pregnancy and cumulative pregnancy rate in the patients with displaced window of implantation more than the receptive group $19(57.7 \%)$ versus $5(41.6 \%)$ and $27(81,8 \%)$ versus $6(50 \%)$, but lower implantation rate in the displaced window of implantation group 6/12 (50\%) versus 25/53 (47.2\%) with higher miscarriage rate in the receptive group $2 / 6$ (33.3\%) versus 4/26 (14.8\%), live birth and take home baby rate in the patients with displaced window of implantation 3 babies delivered to the receptive group 3/12 (25\%), 24 babies to the group of displaced window of implantation 24/53 (45.3\%).

Conclusions: Patients with recurrent unexplained implantation failure may benefit from personalized embryo transfer after determining their window of implantations with endometrial receptivity array testing.
\end{abstract}

Keywords: Embryo transfer, Infertility, Implantation failure

\section{INTRODUCTION}

Recurrent implantation failure is situation defined by failure to get pregnancy after transferring of three or more of good quality embryos in 3 or more IVF cycles. ${ }^{1}$ Multiple factors had been attributed to this situation, these factors may be related to the embryos as in chromosomal abnormalities or related to the uterus as mechanical factors which include uterine septum, submucosa fibroids uterine niche following caesarian surgery, intrauterine adhesions or it could be functional factors as chronic endometritis or non-receptive endometrium. ${ }^{2-6}$ Tubal factors also may be the reason for the recurrent implantation failure in case of communicating hydrosalpinx. ${ }^{7}$ To a lesser extent, thrombophilia and immune factors has to be excluded as it may affect the implantation., 8

Endometrial receptivity is at its peak during the window of implantation which could vary from 4-5 days and was 
thought that it is the same in all patients but actually and according to different studies it varies from patient to patient. ${ }^{10}$ In IVF cycles endometrial receptivity was determined by morphological appearance of the endometrium on transvaginal ultrasound as the endometrial thickness, pattern and blood flow which is not accurate $100 \% .{ }^{11}$ Histological and biochemical markers were used to determine the endometrial receptivity but it did not prove accuracy. ${ }^{12,13}$

Recently genetic testing of the endometrial tissue could determine the gene expression in each phase of endometrium as it was proven that gene expression in each phase could determine the metabolic activity, cellular, humoral immunity, blood coagulation, meiosis and mitotic activity in the endometrial tissue that determine the phase of receptivity. ${ }^{14}$ Gene expression in the endometrial phases could be different in the 7 phases of endometrium (menstrual, early-proliferative, mid-proliferative, lateproliferative, early-secretory, mid-secretory and latesecretory) and vary from up regulation to down regulation according to each phase. ${ }^{15}$ As these activity define the window of implantation we can see that the early secretory phase is associated with pre-receptive endometrium, mid secretory associated with receptive endometrium and late secretory associated with post-receptive endometrium. ${ }^{16}$ This was done through analysing the expression of 248 gene selected for their endometrial receptivity profile using Next generation sequencing in conjunction with bioinformatics tool that gives endometrial receptivity diagnosis. ${ }^{17}$ Studying the gene expression in the endometrial tissue to determine the receptivity was first published by Diaz-Gimeno et al 2011 and its clinical application on patients with recurrent implantation failure was demonstrated by Ruiz-Alonso et al 2013. ${ }^{18,19}$

In patients with recurrent implantation failure eliminating the factors related to embryos and exclusion of anatomical and mechanical uterine and tubal factors beside the full clearance of thrombophilia and immunological factors could categories the patient under unexplained implantation failure which needs to focus on endometrial receptivity using the gene expression testing of endometrial tissue to determine window of implantation through ERA (endometrial receptivity analysis) test. Different studies have demonstrated the displacement of endometrial tissue in patients with recurrent implantation failure with percentage vary from $25-40 \%$ but the clinical application of personalized embryo transfer in patient with displacement of window of implantation and the success of getting clinical pregnancy and live birth after this procedure is the only way to prove its effectiveness. ${ }^{20}$

In the era of personalized medicine which started in the IVF by personalized ovarian stimulation protocol and type of trigger followed by technique of fertilization and mode of luteal phase support, using personalized embryo transfer protocol to gain some success and to help that category of patients to have pregnancy and live births. The aim of our study was to evaluate the clinical application of personalized embryo transfer according to ERA test in patients with unexplained recurrent implantation failure whose endometrium shows displacement of the window of implantation.

\section{METHODS}

A retrospective study was carried out in large IVF center in Abu Dhabi in period from 2017-2021. Patients included in the study were infertile patients with age limit of 43 years old with history of IVF failure after multiple embryo transfer; those patients have extensive work up that excluded hydrosalpinx by hystrosalpingo-graphy and normal uterine cavity through hysteroscopy, normal thrombophilia profile, no immunological factors could be detected.

Exclusion criteria included patients with hydrosalpinx, patients with abnormal cavity, patients with thin endometrium and patients with abnormal embryos on chromosomal study.

All the patients had ERA test to determine the endometrial receptivity timing and window of implantation for each patient, then they had frozen embryo transfer of Euploid high grade embryos obtained through stimulated cycle of each patient (self-oocytes) according to ERA test results.

Patients have been counselled and consented for the procedure, expected outcome, possible complication and possible failure of the transfer even with ERA test.

Study design included retrospective analysis of outcome of personalized embryo transfer of euploid high grade embryos in patients with recurrent implantation failure who had window of implantation diagnosed according to ERA test.

\section{ERA test}

Patients attended the clinic in their second or third day of the period to have transvaginal scan of the uterus and ovaries in order to exclude abnormalities in the ovaries like ovarian cysts and to ensure endometrial shedding, then to start on estradiol valerate $2 \mathrm{mg}$ three times daily, patients continued on estradiol tablets for 7 days and came back for transvaginal scan of the endometrium, when it reached 8 $\mathrm{mm}$, patient started on progesterone suppositories at a dose of $400 \mathrm{mg}$ three times daily and after 5 days endometrial tissue biopsy for ERA test took place. If by day 8 patient did not have the desired endometrial thickness patient was started on estraderm patches $100 \mathrm{mcg}$ to be changed every third day for another 5 days then scan for endometrium thickness before starting the progesterone.

\section{Endometrial tissue biopsy}

Patient were placed in sterile room in dorsal lithotomy position comfortably, Cusco speculum placed in the vagina, cervix to be washed then under ultrasonic guidance 
endometrial tissue sampling using endometrial Pipelle to be done. The sample obtained was placed in tube provided by the manufacture containing RNA stabilizing solution, care was taken to have proper volume of the tissue as if less amount the sample was not enough to be analyzed and if more RNA degeneration occured, the tube was placed in the refrigerator till transferred.

A total 248 gene expression was analyzed through next generation sequencing then adjusted to computerized analyzer to determine the endometrial receptivity.

\section{Results interpretation}

The results were available in two to three weeks. It was either receptive endometrium, early receptive, late receptive, pre-receptive and post-receptive.

In case of receptive endometrium, the embryo transfer was done on the same time we obtained the biopsy. In case early receptive we delayed the transfer by about 12 hours and no need for other biopsy, but in late receptive endometrium we transfered early by 12 hours and no need to repeat endometrial biopsy.

In cases of pre receptive and post receptive endometrium we repeated the biopsy according to instructions provided till we reached the receptive state.

\section{Frozen embryo transfer cycles}

All patients went through frozen embryo transfer cycle after stimulated cycle with preimplantation genetic screening of the 24 chromosome analyses through blastocyst biopsy and normal embryos were frozen through embryo vitrification technique.

Patients who had normal embryos attended the clinic on their second or third day of the cycle for transvaginal scan to ensure normal ovaries and shedding endometrium. Patients started on estradiol valerate tablets $2 \mathrm{mg}$ three times per day and came back after one week to evaluate the endometrium, then started on progesterone suppositories if the desired endometrial thickness reached otherwise to start on estraderm skin patches 100 mcg every third day for another 5 days endometrial thickness is accepted before starting the progesterone. Embryo transfer day was scheduled according to results of ERA test.

\section{Embryo transfer}

Patient was placed in the embryo transfer room in the dorsal lithotomy position in the exact day designed by ERA test, confirmation of the patient was done by embryologist before loading the embryos in the transfer catheter then Cusco speculum to be inserted and cervix cleaned and under ultrasonic guidance embryo transfer was done.
Luteal phase supported with estradiol valerate tablets $2 \mathrm{mg}$ four times daily, progesterone suppositories $400 \mathrm{mg} 4$ times daily.

Patients attended the clinic for B-hCG testing after 2 weeks and transvaginal scan after another two weeks.

\section{Approvals}

This study was approved by research and ethical committee for health pulse network enabling to collect and analyze data available from patient's files and reports with reference number REC/2019/P03.

\section{Outcome and statistical analysis of the study}

Pregnancy rate after personalized embryo transfer in patients with recurrent implantation failure diagnosed with displaced window of implantation according to ERA test in relation to the same category of patient who had receptive endometrium according to ERA test. Clinical pregnancy rate (calculated by presence of intrauterine gestational sacs and cardiac activity detected), implantation rate (the rat of intrauterine gestational sac in relation to number of embryos transferred), ongoing pregnancy rate, miscarriage rate and livebirth rate in both groups. All were displayed in numbers and percentages in text, tables and graphs, Chi square calculator used to calculate the significance, significance calculated to be $\mathrm{p}<0.05$.

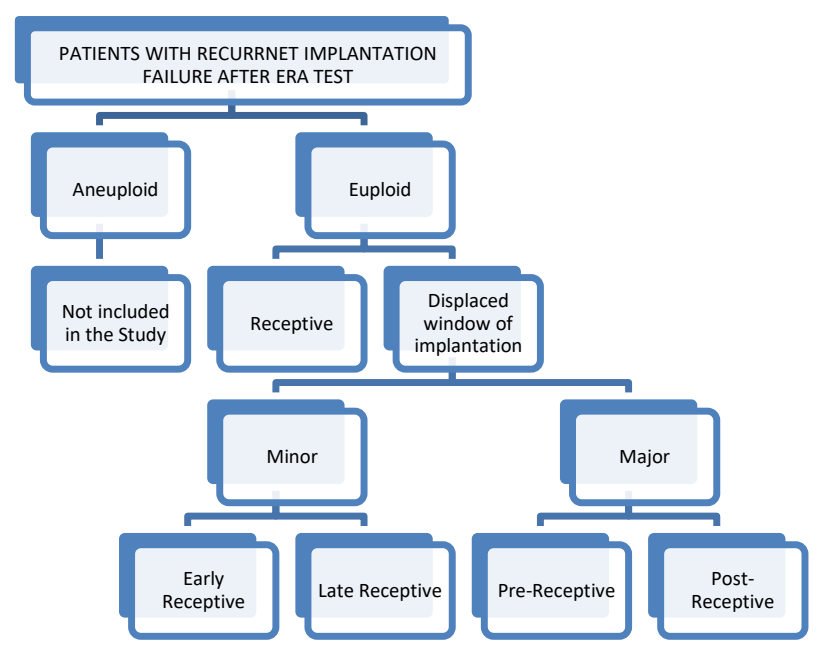

Figure 1: Interpretation of the results.

\section{RESULTS}

This study included 45 patients who had ERA test done due to repeated implantation failure then they had frozen cycle embryo transfer using the same protocol of hormones used in ERA test. Those patients were divided into two major groups. First group included patients with proper receptive endometrium; second group included patients with displaced window of implantation which was 
subdivided into 2 groups, patients with minor displacement which (early and late receptive) and patients with major displacement (pre-receptive and postreceptive).

Table 1: Demographic criteria of the patients.

\begin{tabular}{|lll|}
\hline Demographics & Range & Mean \pm SD \\
\hline Age (years) & $24-43$ & $34.128 \pm 1.856$ \\
\hline BMI (kg/m ( $^{2}$ & $19-38$ & $26.755 \pm 1.164$ \\
\hline $\begin{array}{l}\text { Period of infertility } \\
\text { (years) }\end{array}$ & $3-10$ & $8.866 \pm 3.609$ \\
\hline Previous failed IVF cycles & $4-7$ & $6.32 \pm 2.589$ \\
\hline Previous cycles with PGD & $1-3$ & $1.63 \pm 0.222$ \\
\hline $\begin{array}{l}\text { Previous cycles without } \\
\text { PGD }\end{array}$ & $2-7$ & $4.72 \pm 2.387$ \\
\hline
\end{tabular}

Patients in the first group were 12 patients $(26.7 \%)$, Patients of the second group were $33 / 45$ patients $(73.3 \%)$. Five patients $(11.1 \%)$ with early receptive endometrium, nine patients $(20 \%)$ with late receptive endometrium, seven patients $(15.6 \%)$ with pre-receptive endometrium and 12 patients $(26.7 \%)$ were post-receptive endometrium. The Chi square statistic is 6.6953. The $\mathrm{p}$ value was 0.009667 , significant at $\mathrm{p}<0.05$.

Table 2: Patients included on the study.

\begin{tabular}{|lll|}
\hline Receptive & No. of patients & Percentage \\
\hline $\begin{array}{l}\text { Displaced window } \\
\text { of implantation }\end{array}$ & 33 & 26.7 \\
\hline Chi Statistic & 6.6953 & 73.3 \\
\hline P value & 0.009667 & \\
\hline
\end{tabular}

Table 3: Pregnancy rate.

\begin{tabular}{|lll|}
\hline & No. of patients & Percentage \\
\hline Receptive & 5 & 41.6 \\
\hline $\begin{array}{l}\text { Displaced window } \\
\text { of implantation }\end{array}$ & 19 & 57.7 \\
\hline Chi Statistic & 4.14 & \\
\hline
\end{tabular}

Pregnancy after euploid frozen embryo transfer were 24 pregnancies in total from first embryo transfer in 45 patients $(53.3 \%), 5$ pregnancies in the receptive group $5 / 12$ (41.6\%) and 5/24 (20.8\%) of all pregnancies, 19/33 $(57.7 \%)$ pregnancies in the group of displaced window of implantation The Chi square statistic was 4.14 . The $p$ value was 0.041881 , significant at $\mathrm{p}<0.05 .2$ pregnancies in the early receptive group $2 / 5(40 \%)$ and $2 / 24(0.083 \%)$ of all pregnancies, 6 pregnancies in the late receptive group $6 / 9(66.7 \%)$ and $6 / 24(25 \%)$ of all pregnancies, 5 pregnancies in the pre-receptive group 5/7(71.4\%) and $5 / 24(20.8 \%)$ of all pregnancies, 6 pregnancies from the post-receptive group $6 / 12(50 \%)$ and $6 / 24(25 \%)$ of all pregnancies.
Cumulative pregnancy rate as we had some patients got pregnant on the second embryo transfer so we got cumulative pregnancy of $33 / 45(73.3 \%) .6$ in the receptive group 6/12 (50\%) and 6/33 of all pregnancies (18.2\%) and $27 / 33$ in the group of displaced windows of implantation $(81.8 \%)$. The Chi square statistic was 4.5683 . The $\mathrm{p}$ value was 0.032569 , significant at $\mathrm{p}<0.05 .2$ in the early receptive group $(40 \%)$ and $2 / 26(0.077 \%)$ of pregnancies in the group of displaced window of implantation, 9 pregnancies in the late receptive group 9/11 (82\%) and $9 / 26(34.6 \%)$ of pregnancies in the group of displaced window of implantation, $7 / 9(77.8 \%)$ of the pre-receptive group and $7 / 26(26.9 \%)$ of pregnancies in the group of displaced window of implantation, in the post-receptive group the cumulative pregnancies were 8 out of 12 $(66.7 \%)$ and $8 / 26(30.8 \%)$ pregnancies in the group of displaced window of implantation.

Table 4: Cumulative pregnancy rate.

\begin{tabular}{|lll|}
\hline Receptive & No. of patients & Percentage \\
\hline $\begin{array}{l}\text { Displaced window } \\
\text { of implantation }\end{array}$ & 6 & 50 \\
\hline Chi Statistic & 4.5683 & 81.8 \\
\hline P value & 0.032569 & \\
\hline
\end{tabular}

The miscarriages were 6 miscarriage $6 / 24(25 \%), 2$ in the receptive group $2 / 12(16.7 \%)$ and $2 / 6$ pregnancies $(33.3 \%), 4$ in the group of displaced window of implantation $4 / 33(12.1 \%)$ and $4 / 27(14.8 \%)$ of the pregnancies in this group. The Chi square statistic was 3.9343 . The $\mathrm{p}$ value was 0.047311 , significant at $\mathrm{p}<0.05$. 2 of them in the late receptive group $2 / 9(22.2 \%)$, one in the pre-receptive group $(14.3 \%)$ and one in the post receptive group (12.5\%).

Table 5: Miscarriages.

\begin{tabular}{|lll|}
\hline Parameters & No. of patients & Percentage \\
\hline Receptive & 2 & 33.3 \\
\hline $\begin{array}{l}\text { Displaced window } \\
\text { of implantation }\end{array}$ & 4 & 14.8 \\
\hline Chi statistic & 3.9343 & \\
\hline P value & 0.047311 & \\
\hline
\end{tabular}

Table 6: Implantation rate.

\begin{tabular}{|lll|}
\hline Parameters & No. of patients & Percentage \\
\hline Receptive & 6 & 50 \\
\hline $\begin{array}{l}\text { Displaced window } \\
\text { of implantation }\end{array}$ & 25 & 47.2 \\
\hline Chi statistic & 9.8925 & \\
\hline P value & 0.00166 & \\
\hline
\end{tabular}

Implantation rate was $63,1 \%$ as we got 41 intrauterine sacs with fetal cardiac activity out of 65 embryos transferred, 6 sacs out of 12 embryos transferred in the receptive group 
$(50 \%)$, in the group of displaced windows of implantation we have 25 intrauterine sacs out of 53 embryos transferred (47.2\%). The Chi square statistic was 9.8925. The $\mathrm{p}$ value was 0.00166 , significant at $\mathrm{p}<0.05$, with 2 sac the early receptive out of 5 embryos (40\%), 10 sacs out of 16 embryos in the late receptive group $(62.5 \%), 10$ sacs out of 16 embryos in the pre-receptive group (62.5\%), 13 sacs out of 16 embryos in the post-receptive group (81.3\%).

In our study we got 27 live birth $27 / 55$ (49\%) of the embryos transferred, 3 babies delivered to the receptive group $3 / 12(25 \%), 24$ babies to the group of displaced windows of implantation, 24/53 (45, 3\%) The Chi square statistic was 5.7439 . The $\mathrm{p}$ value was 0.016546 , significant at $\mathrm{p}<0.05$.

With 2 babies for early receptive group, 9 babies for late receptive group, 8 babies for the pre-receptive group and 5 babies for the post receptive group. We had 3 sets of twins and one set of triplets who had early neonatal death due to preterm labor at 26 weeks, we still had 5 ongoing pregnancies.

Table 7: Live births.

\begin{tabular}{|lll|}
\hline & No. of patients & Percentage \\
\hline Receptive & 3 & 25 \\
\hline $\begin{array}{l}\text { Displaced window } \\
\text { of implantation }\end{array}$ & 24 & 45.3 \\
\hline Chi Statistic & 5.7439 & \\
\hline P value & .016546 & \\
\hline
\end{tabular}

\section{DISCUSSION}

Recurrent implantation failure was a sad situation that could affect the couples life and led to a lot of disturbances, this situation needed detailed work up to find reasons and treat it to gain intrauterine healthy pregnancy that ended in live birth.

Multiple studies had investigated the situation and declared that embryos contributed to $20-60 \%$ of the causes of recurrent implantation failure, especially in old aged patients. ${ }^{21}$

On the other hand, transfer normal high-grade embryos did not guarantee healthy pregnancy, from her came the name unexplained recurrent implantation failure. Transferring health high grade embryos in the window of implantation could to some extent decreased the incidence of failure. ${ }^{22}$ The challenge of diagnosing the window of implantation have been ended by studying the gene expression in endometrial tissue to determine the receptivity. As per multiple studies on endometrial receptivity array it was found that the window of implantation had been displaced in around $25 \%$ of cases of implantation failure, which implied the necessities to start personalized embryo transfer according to the diagnosed window of implantation according to ERA test and to find its significance on clinical application. ${ }^{23,24}$

We can see that the window of implantation was displaced in our study in around $73 \%$ of patients which was more than seen in study by Ruiz-Alonso et al in 2013 who found the displacement in only $25 \%$ of cases but in our study we decided to use cycles with unexplained recurrent implantation failure that included the transfer of euploid high grade embryos. In our study the privilege of displaced window of implantation was significant despite small number of patients included. This could be changed of larger number of patients was included was bigger.

The only prove of ERA test significance if on personalized embryo transfer we got intrauterine healthy pregnancy and live birth, so in our study after doing personalized embryo transfer in patient with displaced window of implantation versus normal transfer on day 5 in the group with receptive endometrium we got 24 pregnancies from 1st transfer. 5 pregnancies in the receptive group 5/12 (41. 6\%), 5/24 $(20.8 \%)$ of all pregnancies, $19 / 33(57.7 \%)$ pregnancies in the group of displaced windows of implantation the Chi square statistic was 4.14. The $\mathrm{p}$ value was 0.041881 , significant at $\mathrm{p}<0.05$, which was supported in study done by Patel et al 2019 who had high pregnancy rate $72 \%$ but not significant when compared to the pregnancies obtained in the receptive group.

Not only pregnancy but the cumulative pregnancy rate in our study was also significant that we had in total 33 pregnancies following 1 st and 2nd transfer, 6 of them in the receptive group and 27 in the group of displaced windows of implantation, the Chi square statistic was 4.5683. The $p$ value was 0.032569 , significant at $p<0.05$, which also in agreement with Jayesh et al 2019 who had high cumulative pregnancy rate, 24 actually from 9 pregnancies we got from the second transfer we had 4 patients gets twin pregnancy on the second transfer after they delivered in the first cycle, one of them in the late receptive group, two in the post receptive group and one in the pre-receptive group, this eliminated the effect of endometrial scratching effect of endometrial biopsy during the ERA test and proved the value of determining the window of implantation and it could be extended for every embryo transfer which was supported by study of Mahajan in 2015 .

On the other hand, the implantation rate for receptive group was higher than in the group of displaced windows of implantation which entailed problem in the embryo and or the interaction between the embryo and the endometrium despite transferring the embryos in the window of implantation. But we saw that the miscarriage rate was higher in the receptive group $2 / 6$ versus $4 / 27$ and it was statistically significant, the p value was 0.047311 , significant at $\mathrm{p}<0.05$.

The most important and final outcome was the live birth and took home baby which was the parameter that 
measured the success of our management and actually we got $27 / 45(60 \%)$ live births and the take home babies were $24(53.3 \%)$ as we had early neonatal death due to preterm delivery of triplets at 26 weeks which resulted from two transferred embryos, 3 took home babies in the receptive group and 24 live birth with 21 took home babies in the group of displaced window of implantation. All this went with agreement of study done by Tan et al in 2018 and Mahajan in 2015 with high implantation and pregnancy rates compared to patients without personalized embryo transfer but the results in their studies was not significant in contrary to our study which we found it significant. ${ }^{20,25}$ In our study the patients got pregnant after delivering with the same window of implantation detected by ERA test before the first transfer which indicated its persistence for more than 2 years and no need for another ERA test before the next transfer.

Each pregnancy mattered and even single pregnancy was a success, what about 24 child went home with their parents after multiple trials of failed IVF.

The number of patients included in this study and the expenses of ERA test could de limiting factors so larger studies were required to evaluate the management protocol. Lowering the coast of ERA test was mandatory to allow more patients to benefit from its application.

\section{CONCLUSION}

Personalized embryo transfer after detecting the window of implantation through endometrial receptive array could be useful way in patients with unexplained recurrent implantation failure. Each patient with unexplained recurrent implantation failure should have a chance to go through ERA test to determine window of implantation before the next IVF trial to decrease chances of embryo wasting, to optimize the reproductive outcome, to decrease the stress from failed IVF trials and to lower the coast needed to get take home babies after IVF.

Funding: No funding sources

Conflict of interest: None declared

Ethical approval: The study was approved by the Institutional Ethics Committee

\section{REFERENCES}

1. Bashiri A, Halper KI, Orvieto R. (2018) Recurrent implantation failure-update overview on etiology, diagnosis, treatment and future directions. Reprod Biol Endocrinol. 2018;16(1):121.

2. Voullaire L, Collins V, Callaghan T, McBain J, Williamson R, Wilton L. High incidence of complex chromosome abnormality in cleavage embryos from patients with repeated implantation failure. Fertil Steril. 2007;87(5):1053-8.

3. Fatemi HM, Kasius JC, Timmermans A, Disseldorp JV, Fauser BC, Devroey P, et al. Prevalence of unsuspected uterine cavity abnormalities diagnosed by office hysteroscopy prior to in vitro fertilization. Hum Reprod. 2010;25(8):1959-65.

4. Demirol A, Gurgan T. Effect of treatment of intrauterine pathologies with office hysteroscopy in patients with recurrent IVF failure. Reprod BioMed Online. 2004;8(5):590-4.

5. NIH U. S. National Library of Medicine. Diagnosis of chronic endometritis in endometrial fluid using molecular techniques to improve the outcomes of assisted reproductive treatments. 2017.

6. Shapiro BS, Daneshmand ST, Garner FC, Aguirre M, Hudson C, Thomas S. Evidence of impaired endometrial receptivity after ovarian stimulation for in vitro fertilization: a prospective randomized trial comparing fresh and frozen-thawed embryo transfer in normal responders. Fertil Steril. 2011;96(2):344-8.

7. Ho Y, Chen H, Huang C, Lee C, Lin P, Lee M, Lee T. Peripheral CD56(+) CD16(+) NK cell populations in the early follicular phase are associated with successful clinical outcomes of intravenous immunoglobulin treatment in women with repeated implantation failure. Front. Endocrinol. 2020;21:937.

8. Azem F, Many A, Ami IB, Yovel I, Amit A, Lessing $\mathrm{JB}$, et al. Increased rates of thrombophilia in women with repeated IVF failures. Hum Reprod. 2004;19(2):368370.

9. Sacks G, Yang Y, Gowen E, Smith S, Fay L, Chapman M. Detailed analysis of peripheral blood natural killer cells in women with repeated IVF failure. Am J Reprod Immunol. 2012;67(5):43-42.

10. Lessey BA. Assessment of endometrial receptivity. Fertil Steril. 2011;96(3):522-9.

11. Bonilla-Musoles F, Raga F, Osborne NG, Castillo JC, Bonilla F. Endometrial receptivity: Evaluation with ultrasound. Ultrasound Q. 2013;29(1):3-20.

12. Murray MJ, Meyer WR, Zaino RJ, Lessey BA, Novotny DB, Ireland K, et al. A critical analysis of the accuracy, reproducibility, and clinical utility of histologic endometrial dating in fertile women. Fertil Steril. 2004;81(5):1333-43.

13. Cavagna M, Mantese JC. Biomarkers of endometrial receptivity-a review. Placenta. 2003;24:39-47.

14. Díaz-Gimeno P, Ruíz-Alonso M, Blesa D, Simón C. Transcriptomics of the human endometrium. Int J Dev Biol. 2014;58(2-4):127-37.

15. Ponnampalam AP, Weston GC, Trajstman AC, Susil B, Rogers PA. Molecular classification of human endometrial cycle stages by transcriptional profiling. Mol Hum Reprod. 2004;10(12):879-93.

16. Talbi S, Hamilton AE, Vo KC, Tulac S, Overgaard MT, Dosiou C, et al. Molecular phenotyping of human endometrium distinguishes menstrual cycle phases and underlying-biological-processes-in-normoovulatory-women. 2006;147(3):1097-121.

17. Mirkin S, Nikas G, Hsiu JG, Díaz J, Oehninger S. Gene expression profiles and structural/functional features of the peri-implantation endometrium in natural and gonadotropin-stimulated cycles. J Clin Endocrinol Metab. 2004;89(11):5742-52. 
18. Díaz-Gimeno P, Horcajadas JA, Martínez-Conejero JA, Esteban FJ, Alamá P, Pellicer A, et al. A genomic diagnostic tool for human endometrial receptivity based on the transcriptomic signature. Fertil Steril. 2011;95(1):50-60.

19. Ruiz-Alonso M, Blesa D, Díaz-Gimeno P, Gómez E, Fernández-Sánchez $\mathrm{M}$, Carranza $\mathrm{F}$, et al. The endometrial receptivity array for diagnosis and personalized embryo transfer as a treatment for patients with repeated implantation failure. Fertil Steril. 2013;100(3):818-24.

20. Mahajan N. Endometrial receptivity array: clinical application. J Hum Reprod Sci. 2015;8(3):121-9.

21. Pehlivan T, Rubio C, Rodrigo L, Romero J, Remohi J, Simón C, et al. Impact of preimplantation genetic diagnosis on IVF outcome in implantation failure patients. Reprod Biomed Online. 2003;6(2):232-7.

22. Scott RT, Upham KM, Forman EJ, Hong KH, Scott $\mathrm{KL}$, Taylor D, et al. Blastocyst biopsy with comprehensive chromosome screening and fresh embryo transfer significantly increases in vitro fertilization implantation and delivery rates: a randomized controlled trial. Fertil Steril. 2013;100(3):697.

23. Hashimoto $\mathrm{T}$, Koizumi M, Doshida M, Toya M, Sagara E, Oka N, et al. Efficacy of the endometrial receptivity array for repeated implantation failure in Japan: A retrospective, two-centers study. Reprod Med Biol. 2017;16(3):290-6.

24. Patel JA, Patel AJ, Banker JM, Shah SI, Banker MR. Personalized embryo transfer helps in improving in vitro fertilization/ICSI outcomes in patients with recurrent implantation failure. J Hum Reprod Sci. 2019;12(1):59-66.

25. Tan J, Kan A, Hitkari J, Taylor B, Tallon N, Warraich $\mathrm{G}$, et al. The role of the endometrial receptivity array (ERA) in patients who have failed euploid embryo transfers. J Assist Reprod Genet. 2018;35(4):683-92.

Cite this article as: Bahgat NA, Said W.

Personalized embryo transfer after endometrial receptivity array test in patients with recurrent unexplained implantation failure. Int J Reprod Contracept Obstet Gynecol 2022;11:657-63. 